\title{
Risk Factors for Pseudarthrosis After Surgical Site Infection of the Spine
}

\author{
DOUGLAS A. HOLLERN, MD ${ }^{1,2,3}$ BARRETT I. WOODS, MD ${ }^{1,2}$ NEIL V. SHAH, MD, MS, ${ }^{3}$ GREGORY D. \\ SCHROEDER, MD ${ }^{1,2}$ CHRISTOPHER K. KEPLER, MD, MBA, ${ }^{1,2}$ MARK F. KURD, MD ${ }^{1,2}$ I. DAVID KAYE, \\ MD ${ }^{1,2}$ PAUL W. MILLHOUSE, MD, MBA, ${ }^{3}$ BASSEL G. DIEBO, MD ${ }^{3}$ CARL B. PAULINO, MD, ${ }^{3}$ ALAN S. \\ HILIBRAND, MD, ${ }^{1,2}$ ALEXANDER R. VACCARO, MD, PHD, MBA, ${ }^{1,2}$ KRIS E. RADCLIFF, MD ${ }^{1,2}$ \\ ${ }^{1}$ Rothman Institute, Philadelphia, Pennsylvania, ${ }^{2}$ Department of Orthopaedic Surgery, Sidney Kimmel Medical College at Thomas Jefferson University, \\ Philadelphia, Pennsylvania, ${ }^{3}$ Department of Orthopaedic Surgery and Rehabilitation Medicine, State University of New York, Downstate Medical Center, \\ Brooklyn, New York
}

\begin{abstract}
Background: Pseudarthrosis following spinal fusion is a complication that frequently requires revision surgery. Reported rates of pseudarthrosis after surgical site infection (SSI) range from $30 \%$ to $85 \%$, but few studies have identified infection as an independent risk factor for its development. The purpose of this study was to determine the incidence of clinically symptomatic pseudarthrosis in patient who developed SSI following lumbar fusion and to identify factors associated with its development.

Methods: This was a retrospective review of a prospectively collected database. Patients who underwent spinal surgery and developed SSI between January 2005 and March 2015 with a minimum 2-year follow-up were included. Patient-specific and procedural characteristics were recorded. Presence of pseudarthrosis was determined clinically by the treating surgeon and was confirmed radiographically. All those in the Pseudarthrosis group required a revision procedure after the eradication of infection. Univariate and multivariate analyses were conducted as appropriate.

Results: A total of 416 patients were included. Of these, $21(5.0 \%)$ developed symptomatic pseudarthrosis following SSI. In this cohort, multivariate regression showed that age, Charlson Comorbidity Index, male sex, and surgical approach were not significant predictors of pseudarthrosis formation. However, number of levels fused was found to be the leading predictor for pseudarthrosis development (odds ratio [OR], 1.356/level, $P<.001$ ), followed by body mass index $(\mathrm{OR}, 1.083$ point, $P<.009)$ in this cohort. The number of levels fused was found to be a significant predictor of hardware removal (OR, 1.190/level, $P<.001)$. Of the 21 pseudarthrosis cases, $85.7 \%$ found staphylococcal species, of which $27.8 \%$ exhibited methicillin-resistant Staphylococcus aureus.

Conclusions: The number of spinal levels fused and body mass index are independent predictors of pseudarthrosis in patients who develop SSI after spinal fusion.

Level of Evidence: Level 4

Clinical Relevance: This is the first known study to specifically identify risk factors for the development of symptomatic pseudarthrosis.

Complications

Keywords: symptomatic pseudarthrosis, risk factors, spinal fusion
\end{abstract}

\section{INTRODUCTION}

Spinal surgery is associated with many serious complications, including pseudarthrosis, neurological injury, paralysis, sepsis, and even death. One of the major problems after spinal surgery is development of surgical site infection (SSI). The literature reports $^{1-7}$ the incidence of infection following spinal surgery to be between $0.5 \%$ and $7 \%$. Significant scientific effort has been exerted to identify risk factors that may predispose patients to infection. Several patient-specific factors such as age, body mass index (BMI), subcutaneous fat thickness, and nutritional status have been implicated, in addition to extrinsic factors such as volume of allogenic transfusion and length of surgery. ${ }^{8-10}$ Interventions such as the intraoperative application of vancomycin powder to the surgical site and use of negativepressure dressings have improved infection rates following spinal surgery. ${ }^{11-13}$ Despite these advancements, infections following spinal procedures continue to present a significant challenge to surgeons and serve as a cause of significant morbidity for patients. In addition to the morbidity, 
the financial implications of infection and revision surgery cannot be overstated.

The ultimate goal in management of postoperative spinal infections is to eradicate the pathogen without compromising patient function. Aggressive irrigation and debridement of the wound bed with supplemental antibiotic treatment is the standard of care for all deep and most superficial infections. In each case, the surgeon must decide whether to remove existing spinal instrumentation. Hardware retention is typically achievable in cases where the infection is identified early, appropriate antibiotics are started, and the wound is aggressively debrided. However, certain organisms, such as Staphylococcus aureus, coagulase-negative staphylococci, and Pseudomonas aeruginosa, possess the ability to create adherent biofilms on implanted instrumentation, typically necessitating hardware removal for successful treatment. Early hardware removal, aggressive debridement of bone graft, and the physiological implications of infection can all contribute to the development of pseudoarthrosis. $^{14}$

Reported rates of pseudarthrosis after deep spinal wound infections range from $30 \%$ to $85 \%$ in the literature, but few studies have identified independent risk factors for the development of pseudarthrosis following infection. ${ }^{15}$ The purpose of this study was to determine the incidence of symptomatic pseudarthrosis in a cohort of patients who developed SSI after spinal fusion and to identify specific factors that may increase the risk of this complication. In addition, we evaluated whether removal of hardware in the setting of infection resulted in higher rates of pseudarthrosis. To our knowledge, this is the single largest study to date of pseudarthrosis in the setting of infection.

\section{MATERIAL AND METHODS}

\section{Study Population}

A prospectively collected database at a single institution was retrospectively reviewed to identify all patients who underwent spinal surgery and developed a SSI, as defined by National Healthcare Safety Network criteria, between January 2005 and March 2015. ${ }^{16}$ Patients who underwent spinal procedures (instrumented decompression and fusion) for trauma, degenerative pathologies, and deformity were included.

\section{Patient Demographics}

Clinical data regarding each patient were collected using operative records, billing code queries, patient charts, and clinic notes. Patient characteristics recorded included age, sex, BMI, tobacco use, and Charlson Comorbidity Index (CCI) score.

\section{Surgical Data}

Surgical data collected included number of levels fused, duration of surgery, approach, time until development of infection, number of irrigation and debridement procedures, organisms identified in intraoperative specimens, removal of hardware, presence or absence of radiographic and/or clinical/symptomatic pseudarthrosis, and use of vacuum-assisted closure. Pseudarthrosis was determined clinically by the treating surgeon and confirmed radiographically.

All patients included in the Pseudarthrosis group required an instrumented revision procedure following eradication of infection. All patients received prophylactic antibiotic treatment per the standard of care. Microbiologic cultures were collected from all patients who developed deep SSI.

\section{Statistical Methods}

Univariate analysis was conducted to compare patients who developed pseudarthrosis after infection with those who did not. Variables included in the analysis were type of surgery, number of spinal levels fused, number of washouts needed for infection control, whether removal of hardware was required, timing of hardware removal (if applicable), whether vacuum-assisted closing devices were used, and pathogens identified in culture.

Multivariate analysis was performed on $\mathrm{R}$ version 3.2.2 (Lucent Technologies, Murray Hill, New Jersey) using a Random Forest technique, including a $\chi^{2}$ test, to account for the large number of patient-related factors included in the data analysis and identify potential risk factors for pseudarthrosis. Multiple logistic regression analysis was also performed to determine risk factors for the development of pseudarthrosis, with pseudarthrosis as the categorical dependent variable and the following outcomes/factors as independent variables: age, BMI, sex, CCI number of spinal levels fused, removal of hardware, and surgical approach.

Data management and statistical analysis were performed using Excel 2010 and SPSS version 20 
Table 1. Univariate analysis comparison of demographic information between patients who developed pseudarthrosis and those who did not.

\begin{tabular}{lccc}
\hline & Pseudarthrosis $(\mathbf{n}=\mathbf{2 1})$ & No Pseudarthrosis $(\mathbf{n}=\mathbf{3 9 5})$ & $\boldsymbol{P}$ Value* \\
\hline Sex: n (\%) & & & \\
$\quad$ Male & $7(33.3)$ & $231(58.5)$ & .353 \\
Female & $14(66.7)$ & $164(41.5)$ & .353 \\
Age, y, mean (range) & $56.52(28-83)$ & $57.57(17-87)$ & .943 \\
BMI, mean (range) & $31.50(21.3-51.5)$ & $30.50(14.8-63)$ & .017 \\
CCI, mean (range) & $1.07(0-4)$ & $1.16(0-13)$ & .648 \\
\hline
\end{tabular}

Abbreviations: BMI, body mass index; CCI, Charlson Comorbidity Index.

* Bold indicates significance $(P<.05)$.

(IBM Corp, Armonk, New York). Results were considered statistically significant when $P \leq .05$.

\section{RESULTS}

\section{Demographics and Clinical Data}

A total of 416 patients were included, consisting of 238 men $(57.2 \%)$ and 178 women $(42.9 \%)$ who developed surgical site infection following spinal fusion. Mean age of included patients was 57.1 years (range, 17-87 years). Of 416 patients, 21 (5.0\%) developed a clinically apparent pseudarthrosis (Pseudarthrosis group). More detailed information about patient-specific and procedural characteristics can be found in Table 1 and Table 2, respectively. Of the individuals with available BMI values, 112 $(30 \%)$ patients were overweight, $82(22 \%)$ were obese, and $98(26.3 \%)$ were morbidly obese. There were $81(21.7 \%)$ individuals with a BMI $<25$, of whom only $5(1.3 \%)$ were underweight.

The average CCI did not vary significantly between groups: For patients with pseudarthrosis after infection, mean CCI was 3.57 (range, 0-11), compared with 3.30 for patients without pseudarthrosis (Nonpseudarthrosis group; range, 0-17; $P=.648)$. Among Pseudarthrosis patients, 15 cultures were monomicrobial, whereas 6 patients had polymicrobial culture results. More detailed characteristics of the patients who developed pseudarthrosis can be found in Table 3 .

Aside from spinal fusions involving the lumbar segment (256 patients, 61.5\%), all remaining curves were equally affected (cervical: 140 patients, 33.7\%; thoracic: 146 patients, $35.1 \%$; and sacral: 113 patients, $27.2 \%$ ). Surgeries on multiple segments were slightly more frequent than unisegmental fusions (221 patients, $53.1 \%$ versus 195 patients, $46.1 \%$, respectively). Almost half of the fusions involved 2 segments only (cervicothoracic: 66 patients, $15.9 \%$; thoracolumbar: 40 patients, 9.6\%; and lumbosacral: 98 patients, $23.6 \%$; total $=204$ individuals, $49 \%$ ), whereas surgeries of $\geq 3$ spinal curves were rare (17 patients in total, $4 \%$; of whom only one individual underwent an entire cervicothoraco-lumbo-sacral fusion).

Multivariate regression identified the number of spinal levels fused (odds ratio [OR], 1.356/level, 95\% CI, 1.15-1.54, $P<.001)$ as the most significant predictor for the development of pseudarthrosis, followed by BMI (OR, 1.083/point, 95\% CI, 1.02-1.15, $P=.008$; Figures 1 and 2). Age, CCI, sex, and surgical approach were not found to be significant predictors $(P>.05)$. Timing of hardware removal was not significantly associated

Table 2. Univariate analysis of procedural parameters comparing those who developed pseudarthrosis with those who did not.

\begin{tabular}{|c|c|c|c|c|}
\hline & Pseudarthrosis $(\mathrm{n}=21)$ & No Pseudarthrosis $(n=395)$ & $\chi^{2}$ & $P$ Value* \\
\hline Number of I\&D procedures, mean (range) & $1.48(1-3)$ & $1.29(1-4)$ & & .129 \\
\hline Removal of hardware, $\mathrm{n}(\%)$ & $7(33.3)$ & $42(10.6)$ & 3.95 & .049 \\
\hline Type of procedure, n (\%) & & & $\ldots$ & \\
\hline Fusion & $19(90.4)$ & $318(80.5)$ & & .249 \\
\hline Re-fusion & $1(4.8)$ & $35(8.9)$ & & .517 \\
\hline Laminectomy & $1(4.8)$ & $43(10.9)$ & & .376 \\
\hline Fused levels, n (\%) & $6.24(95 \%$ CI, 4.28-8.20) & $3.36(95 \%$ CI, 3.09-3.63) & 12.6 & .001 \\
\hline 0 & $0(0)$ & $39(9.4)$ & & \\
\hline $1-2$ & $6(1.4)$ & $150(36.1)$ & & \\
\hline $3-4$ & $2(0.5)$ & $96(23.1)$ & & \\
\hline$\geq 5$ & $13(3.1)$ & $110(26.4)$ & & \\
\hline
\end{tabular}

Abbreviation: I\&D, incision and drainage.

$*$ Bold indicates significance $(P<.05)$. 
Table 3. Patients with pseudarthrosis.

\begin{tabular}{|c|c|c|c|c|c|c|c|c|c|}
\hline Patient & Sex & Age, $y$ & Fusion & $\begin{array}{l}\text { No. of Levels } \\
\text { Fused }\end{array}$ & Approach & $\begin{array}{l}\text { No. of I\&D } \\
\text { Procedures }\end{array}$ & $\begin{array}{l}\text { Removal of } \\
\text { Hardware }\end{array}$ & $\begin{array}{c}\text { Mono- vs } \\
\text { Polymicrobial }\end{array}$ & Pathogens \\
\hline 1 & $\mathrm{~F}$ & 55 & Thoracic & 9 & Posterior & 3 & No & Monomicrobial & MSSA \\
\hline 2 & $\mathrm{~F}$ & 60 & Cervical & 1 & Posterior & 1 & Yes & Monomicrobial & Pseudomonas aeruginosa \\
\hline 3 & $\mathrm{M}$ & 49 & Cervical, thoracic & 6 & Combined & 1 & Yes & Monomicrobial & MSSA \\
\hline 4 & $\mathrm{~F}$ & 70 & Cervical, thoracic & 14 & Combined & 1 & No & Polymicrobial & $\begin{array}{l}\text { Enterococcus faecalis Group D } \\
\text { Bacteroides fragilis } \\
\text { CoNS }\end{array}$ \\
\hline 5 & $\mathrm{~F}$ & 40 & Cervical, thoracic & 5 & Posterior & 1 & No & Polymicrobial & $\begin{array}{l}\text { MSSA } \\
\text { CoNS }\end{array}$ \\
\hline 6 & $\mathrm{~F}$ & 66 & Cervical, thoracic & 1 & Anterior & 1 & No & Monomicrobial & MSSA \\
\hline 7 & $\mathrm{M}$ & 41 & Cervical, thoracic & 8 & Combined & 2 & No & Monomicrobial & MRSA \\
\hline 8 & $\mathrm{M}$ & 50 & Lumbar, sacral & 8 & Combined & 1 & Yes & Monomicrobial & MSSA \\
\hline 9 & $\mathrm{~F}$ & 65 & Cervical & 10 & Combined & 1 & No & Monomicrobial & Hafnia alvei \\
\hline 10 & $\mathrm{~F}$ & 59 & Lumbar & 10 & Posterior & 1 & Yes & Monomicrobial & MRSA \\
\hline 11 & M & 61 & Lumbar & 6 & Posterior & 1 & Yes & Monomicrobial & MRSA \\
\hline 12 & M & 28 & Lumbar, sacral & 6 & Posterior & 1 & Yes & Monomicrobial & MRSA \\
\hline 13 & M & 55 & Lumbar & 2 & Combined & 3 & No & Polymicrobial & $\begin{array}{l}\text { Citrobacter koseri } \\
\text { B. fragilis } \\
\text { Eggerthella lentum } \\
\text { Corynebacterium spp. }\end{array}$ \\
\hline 14 & $\mathrm{~F}$ & 64 & Cervical, thoracic & 3 & Posterior & 2 & No & Polymicrobial & $\begin{array}{l}\text { Corynebacterium spp. } \\
\text { Proteus mirabilis } \\
\text { CoNS }\end{array}$ \\
\hline 15 & $\mathrm{~F}$ & 57 & Lumbar & 11 & Combined & 1 & No & Monomicrobial & MSSA \\
\hline 16 & $\mathrm{M}$ & 55 & Cervical, thoracic & 2 & Combined & 3 & No & Polymicrobial & $\begin{array}{l}\text { C. koseri } \\
\text { B. fragilis } \\
\text { E. lentum } \\
\text { Corynebacterium spp. }\end{array}$ \\
\hline 17 & $\mathrm{~F}$ & 73 & Thoracic & 3 & Posterior & 2 & Yes & Monomicrobial & MRSA \\
\hline 18 & $\mathrm{~F}$ & 64 & Lumbar, sacral & 1 & Posterior & 1 & No & Monomicrobial & MSSA \\
\hline 19 & $\mathrm{~F}$ & 83 & Cervical & 2 & Posterior & 2 & No & Monomicrobial & MSSA \\
\hline 20 & $\mathrm{~F}$ & 40 & Thoracic, lumbar & 5 & Posterior & 1 & No & Polymicrobial & $\begin{array}{l}\text { MSSA } \\
\text { CoNS }\end{array}$ \\
\hline 21 & $\mathrm{~F}$ & 46 & Lumbar, sacral & 16 & Combined & 1 & No & Monomicrobial & MSSA \\
\hline
\end{tabular}

Abbreviations: CoNS, coagulase-negative staphylococci; I\&D, incision and drainage; MRSA, methicillin-resistant Staphylococcus aureus; MSSA, methicillin-sensitive S. aureus.

with development of pseudarthrosis $(P=.967)$. The number of levels fused was found to be a statistically significant predictor of hardware removal (OR, 1.19/level, 95\% CI, 1.08-1.30, $P<.001 ;$ Figure 3).

\section{Pathogen Culture}

Among all 416 patients who developed SSI, cultures showed that 24 pathogens, including 23 bacterial pathogens and 1 fungus, were isolated. Eighteen patients $(4.6 \%)$ either had no cultures taken or showed no growth once cultures resulted.

The prevalence of gram-positive rods in culture did not vary significantly between groups $(80.9 \%$ Pseudarthrosis versus $86.3 \%$ Nonpseudarthrosis, $P=.959$ ); this was also the case for gram-positive cocci $(14.3 \%$ among Pseudarthrosis versus $9.9 \%$ without, $P=.435)$. Rate of identification in culture of gram-negative rods also did not differ significantly between the 2 groups $(28.6 \%$ in those with pseudarthrosis versus $44.3 \%$ in those without,
$P=.324)$. Anaerobes were identified significantly more frequently in patients who developed pseudarthrosis than in those who did not $(14.3 \%$ versus $6.3 \%, P=.01)$.

Among the 21 pseudarthrosis cases, staphylococcal species were the most common etiologic agents of infection among all gram-positive cocci $(18 / 21$ isolates; $85.7 \%$ ), with $27.8 \%$ of these exhibiting methicillin resistance $(5 / 18)$. Of the 395 isolates from patients without pseudarthrosis, staphylococcal species were also the most common isolate among gram-positive cocci and were present in $86.1 \%$ (324/ 395) of positive cultures (including 1 case with polymicrobial culture results). Of these isolates, $26.2 \%(85 / 324)$ were further demonstrated to be methicillin-resistant Staphylococcus aureus; the culture rates were comparable between patients with and without pseudarthrosis $(P>.05)$. Overall, methicillin-sensitive $S$. aureus culture rates did not vary significantly between groups $(42.9 \%$ among Pseudarthrosis versus $30.6 \%$ without, $P>.05$ ). Coagulase-negative staphylococci were the next 


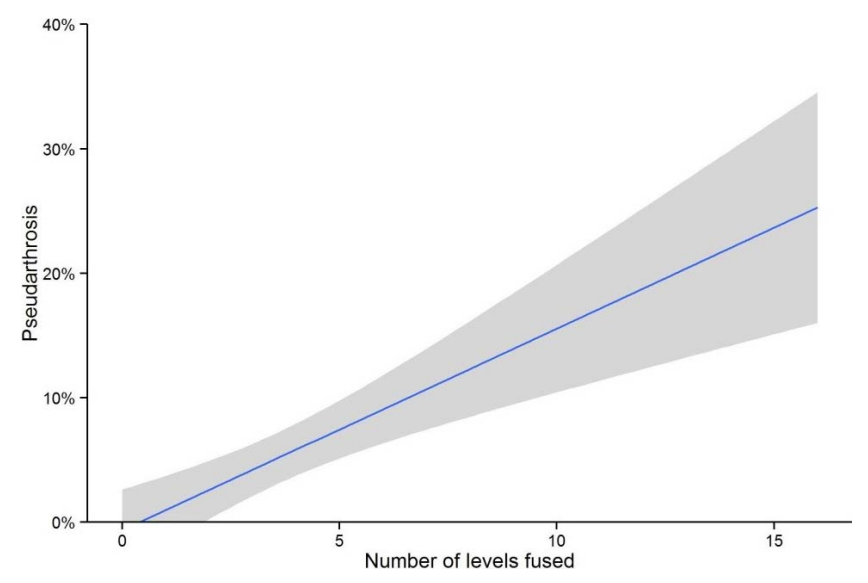

Figure 1. Variable importance plot of the number of levels fused, showing a statistically significant association with the development of pseudarthrosis. The grey shadow spans the $95 \%$ confidence interval region.

most common agent identified in patients without pseudarthrosis, occurring in $30.6 \%$ of nonpseudarthrosis cultures (118/395). More detailed culture results can be found in Table 4 .

\section{DISCUSSION}

Despite the use of modern antibiotics, SSI remains a relatively common postoperative complication. Infections associated with spinal surgery have a large impact on a patient's postoperative course, often requiring additional open procedures; infection may also increase the risk of other complications, such as pseudarthrosis. As previously mentioned, the postoperative infection rate for spinal procedures has been reported in recent literature $^{1-6}$ to range from $0.7 \%$ to $15.0 \%$.

The present study sought to examine the rate of development of pseudarthrosis in patients with postoperative infection after spinal fusion surgery and to identify patient-specific and procedural characteristics that predicted pseudarthrosis development. To our knowledge, this study examined the largest such infection cohort. Comparison of our results with those of previous studies was difficult, given the dearth of literature describing spinal pseudarthrosis in the context of infection. Nonetheless, several illuminating contrasts were highlighted. In 1997, Weiss et $\mathrm{al}^{15}$ reported on the relationship between SSI and the development of pseudarthrosis in a relatively large series. Of 900 patients who underwent a posterolateral lumbar fusion, 29 $(3.2 \%)$ developed SSI. Of these, $11(38 \%)$ were subsequently diagnosed with pseudarthrosis. This was a far higher rate than observed in the present study, which may partly be explained by improve-

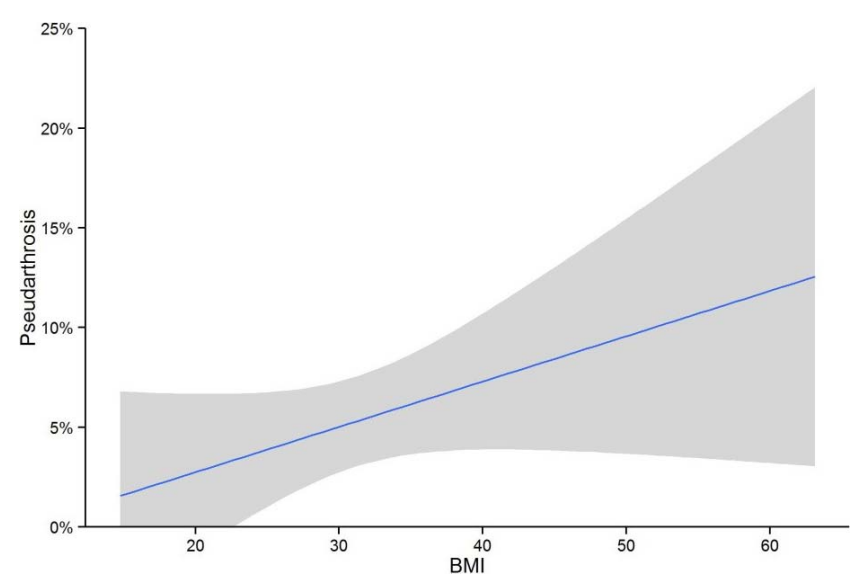

Figure 2. Variable importance plot of body mass index (BMI), showing a statistically significant association with the development of pseudarthrosis. The gray shadow spans the $95 \%$ confidence interval region.

ments in surgical techniques and implant technology with a resultant increase in successful fusions over the past 20 years. ${ }^{17-19}$ The fact that the pseudarthrosis patients in the present study were selected specifically because they were diagnosed first clinically and then confirmed radiographically may also have contributed to this discrepancy, because nearly $30 \%$ of pseudarthroses have been suggested to be asymptomatic. $^{20}$ In addition, in the Weiss et $\mathrm{al}^{15}$ study model, female sex, fusion involving the sacrum, and the use of allografts were significant predictors of pseudarthrosis development. Whereas the present study did not identify female sex as a risk factor for development of pseudarthrosis, the percentage of men in the Nonpseudarthrosis group was nearly twice that of the Pseudarthrosis group. The potential for an association between female sex and pseudarthrosis development merits further study.

The results of the present study also contradicted those of Mok et al, ${ }^{21}$ who performed a small casecontrol study to determine the impact of SSI on clinical outcomes of posterior spinal fusion as measured by patients' Short Form-36 questionnaire scores. Of 16 patients with postoperative infections, 3 underwent reoperation for pseudarthrosis (19\%). The rate of development of pseudarthrosis in the noninfected group did not differ significantly from that of the control group. In addition, none of the variables evaluated-including number of levels fused-were associated with a significant difference in outcomes between the infection group and the control group. However, the number of patients included in the study was very small. Thus, it was possible that associations were missed, which may 
Table 4. Results of microbial cultures taken from all patients included in this study.

\begin{tabular}{|c|c|c|c|}
\hline Category & Pseudarthrosis $(n=21)$, no. $(\%)$ & No Pseudarthrosis $(\mathrm{n}=395)$, no. $(\%)$ & $P$ Value* \\
\hline Gram-positive cocci & $18(85.7)$ & $341(86.3)$ & .959 \\
\hline Staphylococcus aureus & $18(85.7)$ & $324(86.1)$ & \\
\hline MRSA & $5(23.8)$ & $85(21.5)$ & $>.050$ \\
\hline MSSA & $9(42.9)$ & $121(30.6)$ & $>.050$ \\
\hline Other & $4(19.0)$ & 118 (29.9) & \\
\hline Streptococcus spp. & $0(0)$ & $17(4.3)$ & \\
\hline Gram-positive rods & $3(14.3)$ & $36(9.9)$ & .435 \\
\hline Corynebacterium spp. & $3(14.3)$ & $25(6.3)$ & \\
\hline Lactobacillus spp. & $0(0)$ & $2(0.5)$ & \\
\hline Propionibacterium spp. & $0(0)$ & $9(2.3)$ & \\
\hline Gram-negative rods & $6(28.6)$ & $175(44.3)$ & .324 \\
\hline Enterococcus spp. & $1(4.8)$ & $34(8.6)$ & \\
\hline Klebsiella spp. & $0(0)$ & $1(0.3)$ & \\
\hline Pseudomonas spp. & $1(4.8)$ & $0(0)$ & \\
\hline Escherichia coli & $0(0)$ & $32(8.4)$ & \\
\hline Citrobacter spp. & $0(0)$ & $39(9.9)$ & \\
\hline Enterobacter spp. & $2(4.5)$ & $5(1.3)$ & \\
\hline Acinetobacter spp. & $1(4.8)$ & $0(0)$ & \\
\hline Delftia acidovorans (Comamonas) & $0(0)$ & $24(6.1)$ & \\
\hline Fusobacterium varium & $0(0)$ & $2(0.5)$ & \\
\hline Hafnia alvei & $1(4.8)$ & $19(4.8)$ & \\
\hline Haemophilus spp. & $0(0)$ & $2(0.5)$ & \\
\hline Prevotella spp. & $0(0)$ & $13(3.3)$ & \\
\hline Serratia marcescens & $0(0)$ & $4(1.0)$ & \\
\hline Anaerobes & $3(14.3)$ & $25(6.3)$ & .010 \\
\hline Bacteroides spp. & $0(0)$ & $13(3.3)$ & \\
\hline Anaerobic gram positive cocci & $3(14.3)$ & $8(2.0)$ & \\
\hline Clostridium spp. & $0(0)$ & $4(1.0)$ & \\
\hline Fungi & $0(0)$ & $3(0.76)$ & .646 \\
\hline Candida spp. & $0(0)$ & $3(0.76)$ & \\
\hline
\end{tabular}

Abbreviations: MRSA, methicillin-resistant Staphylococcus aureus; MSSA, methicillin-sensitive S. aureus; spp, species.

$*$ Bold indicates significance $(\mathrm{P}<.05)$.

have helped to explain the contradiction with the results of the present study, which identified BMI and number of spinal levels fused as possible predictors of pseudarthrosis development. Chen et $\mathrm{al}^{22}$ conducted a larger cohort study examining long-term treatment outcomes after postoperative wound infection in patients with posterior spinal instrumentation. They found that 10 of 51 patients required implant removal $(19.6 \%)$, with an overall pseudarthrosis rate of $31.4 \%$. The overall hardware

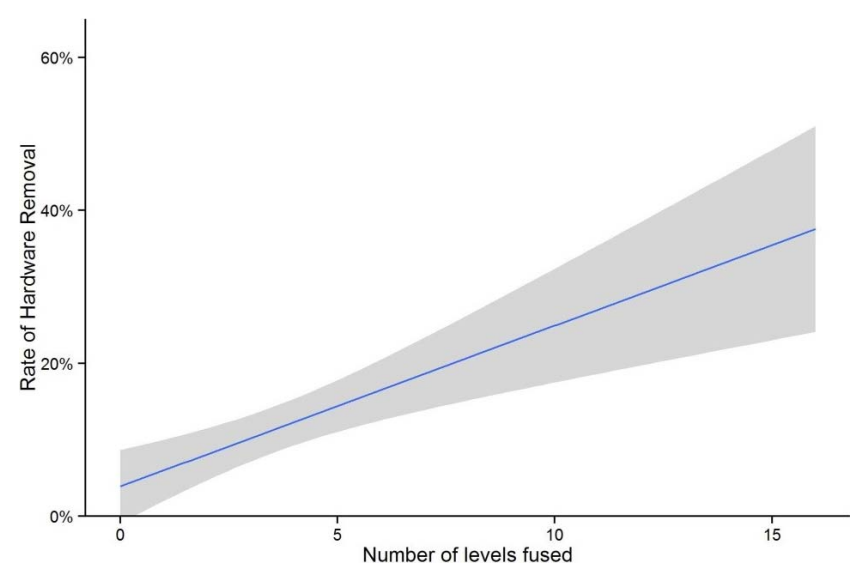

Figure 3. Number of levels fused associated with removal of hardware. The gray shadow spans the $95 \%$ confidence interval region. removal rate was comparable with that of the present study (49/416, or $11.8 \%$ ), but the pseudarthrosis rate was again much higher in the smaller cohort. This was likely attributed to similar factors described above in contrast with the Weiss et $\mathrm{al}^{15}$ cohort.

Etiologic agents of infection were, overall, comparable between patients with and without pseudarthrosis in this study; we identified staphylococcal species to be the most common infectious agents found in pseudarthroses, a finding consistent with multiple other studies. ${ }^{1,6,8,10}$ We were surprised that removal of hardware was not a significant predictor in the multivariate regression model despite showing significance in the univariate analysis. This was likely due to bicorrelation; removal of hardware showed a significant association with the number of levels fused (OR, 1.19/ level). Thus, each additional level included in the fusion operation may increase the risk of complications, such as subsequent requirement of removal of hardware.

Limitations of the present study included inclusion all of surgical approaches in a single group, without the ability to separately analyze the impact 
of approach. Posterior approach has a reported association with lower rates of pseudarthrosis development, and an ideal regression model may have better accounted for this covariate. ${ }^{20}$ In addition, though the sample size was much larger than those of many previous studies, this study identified no impact of sex on pseudarthrosis development, though such an effect has been suggested before. ${ }^{15}$ Comparison of asymptomatic pseudarthrosis patients with both symptomatic pseudarthroses and patients without pseudarthrosis may prove useful in future studies for further evaluation of the effects of female sex. Future studies with additional radiographic analysis would also aid in examining the effects of spinal alignment on patient-reported outcome measures following revision for pseudarthrosis. Finally, none of the aforementioned studies evaluated risk factors for pseudarthrosis following infection in the specific regional context of the lumbar spine. In this study, 2 factors that were most highly associated with the development of pseudarthrosis following infection were the number of levels fused and BMI. The impact of BMI is well documented in the literature, with obesity established as a risk factor for pseudarthrosis. ${ }^{20,23,24}$ However, the interplay between patient-specific factors such as BMI, procedural characteristics such as number of levels fused or approach, and the presence of postoperative infection may vary by involved spinal region. ${ }^{25}$ The present study examined this interplay in the lumbar spine, and future region-specific research should be conducted to examine the specific risks at each level.

\section{CONCLUSION}

In a cohort of unprecedented power with specific focus on patients with clinically apparent pseudarthrosis, multivariate logistic regression analysis identified number of spinal levels fused in the index procedure and BMI as significant predictors of pseudarthrosis development following spinal surgical site infections. Further research is recommended to explore the impact of surgical site infection and inflammation on the subsequent development of pseudarthrosis as well as regional specificity of risk factors.

\section{ACKNOWLEDGMENTS}

The authors would like to thank Susan Lammers for her contributions to this study. This study was approved by the Thomas Jefferson University Institutional Review Board.

\section{REFERENCES}

1. Meng F, Cao J, Meng X. Risk factors for surgical site infections following spinal surgery. $J$ Clin Neurosci. 2015;22(12):1862-1866. https://doi.org/10.1016/j.jocn.2015.03. 065

2. Radcliff KE, Neusner AD, Millhouse PW, et al. What is new in the diagnosis and prevention of spine surgical site infections. Spine J. 2015;15(2):336-347. https://doi.org/10.1016/ j.spinee.2014.09.022

3. Meredith DS, Kepler CK, Huang RC, Brause BD, Boachie-Adjei O. Postoperative infections of the lumbar spine: presentation and management. Int Orthop. 2012;36(2):439-444. https://doi.org/10.1007/s00264-011-1427-z

4. Bible JE, Biswas D, Devin CJ. Postoperative infections of the spine. Am J Orthop (Belle Mead NJ). 2011;40(12):E264E271.

5. Pull ter Gunne AF, Cohen DB. Incidence, prevalence, and analysis of risk factors for surgical site infection following adult spinal surgery. Spine (Phila Pa 1976). 2009;34(13):1422-1428. https://doi.org/10.1097/BRS.0b013e3181a03013

6. Weinstein MA, McCabe JP, Cammisa FP. Postoperative spinal wound infection: a review of 2,391 consecutive index procedures. J Spinal Disord. 2000;13(5):422-426.

7. Wang TY, Back AG, Hompe E, Wall K, Gottfried ON. Impact of surgical site infection and surgical debridement on lumbar arthrodesis: a single-institution analysis of incidence and risk factors. $J$ Clin Neurosci. 2017;39:164-169. https://doi. org/10.1016/j.jocn.2017.01.020

8. Fang A, Hu SS, Endres N, Bradford DS. Risk factors for infection after spinal surgery. Spine (Phila Pa 1976). 2005;30(12):1460-1465. https://doi.org/10.1097/01.brs.0000166 $532.58227 .4 \mathrm{f}$

9. Olsen MA, Nepple JJ, Riew KD, et al. Risk factors for surgical site infection following orthopaedic spinal operations. $J$ Bone Joint Surg Am. 2008;90(1):62-69. https://doi.org/10.2106/ JBJS.F.01515

10. Woods BI, Rosario BL, Chen A, et al. The association between perioperative allogeneic transfusion volume and postoperative infection in patients following lumbar spine surgery. J Bone Jt Surg. 2013;95(23):2105. https://doi.org/10. 2106/JBJS.L.00979

11. Schroeder JE, Girardi FP, Sandhu H, Weinstein J, Cammisa FP, Sama A. The use of local vancomycin powder in degenerative spine surgery [published online August 7, 2015]. Eur Spine J. https://doi.org/10.1007/s00586-015-4119-3

12. Godil SS, Parker SL, O’Neill KR, Devin CJ, McGirt MJ. Comparative effectiveness and cost-benefit analysis of local application of vancomycin powder in posterior spinal fusion for spine trauma: clinical article. J Neurosurg Spine. 2013;19(3):331335. https://doi.org/10.3171/2013.6.SPINE121105

13. Theologis AA, Demirkiran G, Callahan M, Pekmezci M, Ames C, Deviren V. Local intrawound vancomycin powder decreases the risk of surgical site infections in complex adult deformity reconstruction: a cost analysis. Spine (Phila 
Pa 1976). 2014;39(22):1875-1880. https://doi.org/10.1097/ BRS.0000000000000533

14. Kim J Il, Suh KT, Kim S-J, Lee JS. Implant removal for the management of infection after instrumented spinal fusion. $J$ Spinal Disord Tech. 2010;23(4):258-265. https://doi.org/10. 1097/BSD.0b013e3181a9452c

15. Weiss LE, Vaccaro AR, Scuderi G, McGuire M, Garfin SR. Pseudarthrosis after postoperative wound infection in the lumbar spine. J Spinal Disord. 1997;10(6):482-487.

16. Brislin B, Vaccaro AR. Advances in posterior lumbar interbody fusion. Orthop Clin North Am. 2002;33(2):367-374. https://doi.org/10.1016/S0030-5898(01)00013-X. Accessed November 14, 2019.

17. Leven D, Cho SK. Pseudarthrosis of the cervical spine: risk factors, diagnosis and management. Asian Spine J. 2016;10(4):776-786. https://doi.org/10.4184/asj.2016.10.4.776

18. Horan TC, Andrus M, Dudeck MA. CDC/NHSN surveillance definition of health care-associated infection and criteria for specific types of infections in the acute care setting. Am J Infect Control. 2008;36(5):309-332. https://doi.org/10. 1016/j.ajic.2008.03.002

19. Reitman CA, Anderson DG, Fischgrund J. Surgery for degenerative spondylolisthesis: open versus minimally invasive surgery. Clin Orthop Relat Res. 2013;471(10):3082-3087. https://doi.org/10.1007/s11999-013-3171-8

20. Abdu WA, Lurie JD, Spratt KF, et al. Degenerative spondylolisthesis: does fusion method influence outcome? Fouryear results of the spine patient outcomes research trial. Spine (Phila Pa 1976). 2009;34(21):2351-2360. https://doi.org/10. 1097/BRS.0b013e3181b8a829

21. Mok JM, Guillaume TJ, Talu U, et al. Clinical outcome of deep wound infection after instrumented posterior spinal fusion: a matched cohort analysis. Spine (Phila Pa 1976). 2009;34(6):578583. https://doi.org/10.1097/BRS.0b013e31819a827c

22. Chen S-H, Lee C-H, Huang K-C, Hsieh P-H, Tsai S-Y. Postoperative wound infection after posterior spinal instrumentation: analysis of long-term treatment outcomes. Eur Spine J. 2015;24(3):561-570. https://doi.org/10.1007/s00586-014-3636-9

23. Patel N, Bagan B, Vadera S, et al. Obesity and spine surgery: relation to perioperative complications. J Neurosurg Spine. 2007;6(4):291-297. https://doi.org/10.3171/spi.2007.6.4.1

24. Jiménez-Avila JM, García-Valencia J, Bitar-Alatorre WE. Risk factors affecting fusion in the treatment of lumbar spine instability [in Spanish]. Acta Ortop Mex. 25(3):156-160.

25. Tetreault L, Nouri A, Singh A, Fawcett M, Nater A, Fehlings MG. An assessment of the key predictors of perioperative complications in patients with cervical spondylotic myelopathy undergoing surgical treatment: results from a survey of 916 AOSpine international members. World Neurosurg. 2015;83(5):679-690. https://doi.org/10.1016/j. wneu.2015.01.021

Disclosures and COI: No authors had any conflicts of interest or disclosures that have directly or indirectly influenced this study. The following authors have no disclosures: D.A.H., N.V.S., I.D.K., B.G.D. The following authors are consultants for or have received institutional or research support from the following companies: B.I.W.: Altus, NEXXT Spine, Precision Spine, and Stryker; G.D.S.: Advance Medical, AOSpine, Medtronic, Medtronic Sofamor Danek; C.K.K.: Biomet, Medtronic, and Pfizer; M.F.K.: Duratap, Innovative Surgical Designs, and Stryker; P.W.M.: Globus Medical; C.B.P.: DePuy/Johnson \& Johnson/Ethicon; A.S.H.: Amedica, Benvenue, Biomet, Lifespine, Nexgen, Paradigm Spine, PSD, Spinal Ventures, and Vertiflex; A.R.V.: Advanced Spinal Intellectual Properties, Aesculap, Atlas Spine, Avaz Surgical, Bonovo Orthopaedics, Computational Biodynamics, Cytonics, Dimension Orthotics, Electrocore, Elsevier, Flagship Surgical, FlowPharma, Gamma Spine, Gerson Lehrman Group, Globus Medical, Guidepoint Global, Health Point Capital, In Vivo, Innovative Surgical Design, Insight Therapeutics, Medacorp, Medtronic, Nuvasive, Paradigm Spine, Parvizi Surgical Innovations, Prime Surgeons, Progressive Spinal Technologies, Replication Medica, Small Bone Innovations, Spine Medica, SpineWave, Spinology, Springer, Stout Medical, Stryker, Taylor Francis/Hodder \& Stoughton, Thieme, Vertiflex, and Vexim; K.E.R.: 4 Web Medical, Globus Medical, Medtronic, NEXXT Spine, Nuvasive, Orthofix Inc, Orthopedic Sciences Inc, Pacira Pharmaceuticals, Simplify Medical, Stryker, and Zimmer.

Corresponding Author: Douglas A. Hollern, MD, Department of Orthopaedic Surgery and Rehabilitation Medicine, SUNY Downstate Medical Center, 450 Clarkson Ave, MSC 30, Brooklyn, NY 11203. Phone: (718) 221-5270; Email: doughollern@gmail.com.

Published 31 December 2019

This manuscript is generously published free of charge by ISASS, the International Society for the Advancement of Spine Surgery. Copyright (c) 2019 ISASS. To see more or order reprints or permissions, see http://ijssurgery.com. 